\title{
Significance of computed tomography scans in head injury
}

\author{
Suryapratap Singh Tomar, Anuj Bhargava, Nikitha Reddy
}

Narayana Medical College, Nellore, India

Email: dr.suryapratap_singh_tomar@yahoo.com

Received 7 July 2013; revised 8 August 2013; accepted 15 August 2013

Copyright (C) 2013 Suryapratap Singh Tomar et al. This is an open access article distributed under the Creative Commons Attribution License, which permits unrestricted use, distribution, and reproduction in any medium, provided the original work is properly cited.

\section{ABSTRACT}

Background: In view of the growing population and increased vehicle use, traumatic brain injury is becoming the most common and devastating problem especially in young healthy people. It has occupied second place next to cancer as leading cause of death. In this new era of radiology, computed tomography has become the primary modality of choice in the initial assessment of head injury patients as it is widely available, faster and highly accurate in detecting skull fractures and acute intracranial hemorrhage. Aim: It is to demonstrate the importance of ct scanning in the initial treatment planning, follow up and long term management of patients with acute head injury of varying severity. Patients: A retrospective study has been carried out of 100 cases of acute head trauma with positive CT scanning referred to Narayana medical college and hospital, Nellore, India between $30^{\text {th }}$ April 2012 to $1^{\text {st }}$ May 2013 [1 year retrospective study]. Results: The post traumatic intracranial consequences have been collected, reviewed and analyzed, where in $82 \%$ of cases males were involved, and most of the injured people belong to the first three decades of life, the main cause being road traffic accident which is growing in incidence in this $21^{\text {st }}$ century. Most of the cases which were studied showed skull fractures, which indicates that there has been significant trauma and in a single case more than one pathology related to cranium has been seen. The most common post traumatic consequences found in the study of adults are contusions, brain edema and intracerebral hematomas, while other sequelae such as subarachnoid hematomas, subdural hematomas and extradural hematomas are encountered less. Diffuse and focal cerebral edema was being the most common pathology following trauma in children. Conclusion: CT scanning is the primary modality of choice in the diagnostic work up of patients with acute head trauma for identifying the various intracranial consequences following head injury especially within 48 hours which helps in the initial assessment, treatment planning, follow up and long term management of patients.

Keywords: Head Injury; CT Scanning; Hematoma

\section{INTRODUCTION}

Trauma is a one of the most common cause of death and lifelong disability in the early decades of life of which majority of cases are neurological trauma [1].

Various literatures have estimated that nearly 1.6 million cranial injuries occur in the United States each year, resulting in over 50,000 - 60,000 deaths and over 70,000 - 80,000 patients with lifelong neurological deficits [2-4]. The diagnosis and management of head trauma have been changed significantly with the wide availability of ct scan since its inception in 1970s [1,2], this study demonstrates the importance of ct scan in acute head trauma, showing the different intracranial consequences and their frequencies have been correlated with age, sex, and the presence of a skull fracture $[4,5]$.

\section{MATERIAL AND METHODS}

During a 12-month period, between $30^{\text {th }}$ April 2012 to ${ }^{\text {st }}$ May 2013 [1 year retrospective study], randomly selected 100 cases of acute head trauma with positive CT scan findings are studied retrospectively.

The ages are ranging from 1 to 69 years. In each case, the age, and sex of the patient, type of trauma, any associated fracture, and CT sequelae with their percentages are all documented.

\section{RESULTS}

Out of 100 cases included in the study, 82 cases (82\%) are males, while female cases are only 18 cases constituting $18 \%$.

The age distribution is clarified in Figure 1.

An associated fracture is documented in 70 cases 


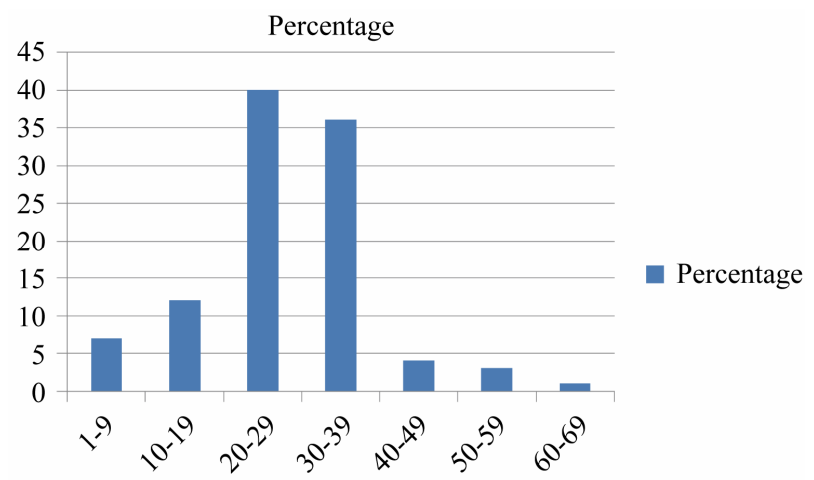

Figure 1. The incidence of acute head trauma in different age groups.

(70\%) of the cases; the non-fractured cases are only 30, constituting 30\%. Regarding the etiologies, road traffic accidents (RTA) is the cause of injury in $89 \%$ of the cases, while fall from heights (FFH), and assault injuries constitute $6 \%$, and $5 \%$ of the etiology, respectively. The incidence of different intracranial sequelae is shown in Figure 2, where a localized brain edema is shown in 66 cases, the other sequelae: i.e.: an intracerebral hematoma in 20 cases, a brain contusion in 22 cases, a subdural hematoma in 18 cases, a diffuse brain swelling in 21 cases, a subarachnoid, epidural, pneumocephalus and an Intraventricular hematoma in 23, 12, 8 and 6 cases respectively.

Other less common sequelae, such as midbrain bleed or edema and intracranial foreign body are encountered less frequently, although not shown in the documentation and charts.

\section{DISCUSSION}

As per the various literatures, RTA is the most common etiology in head injury cases and third and forth decade of life is commonest age group with male gender because they are involved in economic and social life [2-9].

In patients with multiple injuries, head is the common affected part. Also it is found that children and young adults are most commonly involved in trauma [5,6,10].

In our study, there is a decrease in the incidence of head trauma with increasing age after second decade of life. In some other two literatures, pediatric age group constituted 53\% and 39\% respectively $[7,8]$.

This study shows that more than two thirds of the cases are due to road traffic accidents (RTA) constituting $89 \%$ of all the cases, whereas, fall from height (FFH), and assault injuries constitute $6 \%$ and $5 \%$ respectively. The high incidence of RTAs reflects a high contribution to the etiology which is indeed an almost double of that figure found in an old study a 46\% [7].

Various literatures have noted that in children RTA and $\mathrm{FFH}$ are the main causes in head injury, while in

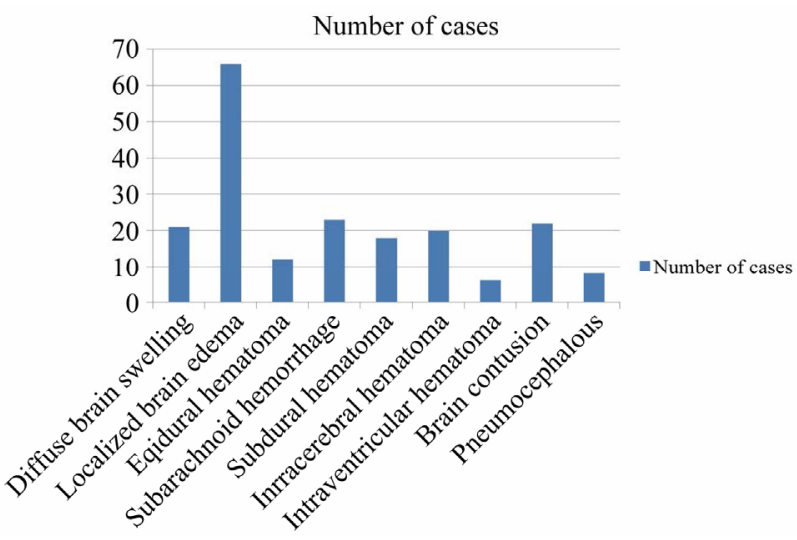

Figure 2. The incidence (number) of various post-injury sequelae of head trauma.

adults assaults are added in main etiology too [4].

In the literature, there are some studies which considered falls as the main cause of acute head trauma [1].

The huge raise in the cases of acute head injuries may be due to high number of vehicles, poor roads, ignorance of the traffic rules and regulations. There is a lot of debate concerning the significance of finding a fracture in the post-traumatic skull radiographs [9], however, no much stress is laid on this point since the majority of the cases were referred immediately for an urgent CT.

In this study $70 \%$ of the cases are associated with fractures, while, the non-fractured cases constitute $30 \%$ only, this high incidence might be explained on the basis of severity and the selectivity of the cases.

A fracture on radiography indicates a significant force has applied to the bony vault.

However, the lack of visualization of a fracture does not exclude a significant injury to the underlying brain; therefore, a skull fracture may or may not indicate a brain parenchyma injury [10,11]. The literature shows an incidence of $2 \%$ - $42 \%$ of fractures in acute head trauma $[4,5,9,12-14]$.

\section{PATHOLOGICAL TYPES OF INTRACRANIAL SEQUELAE SEEN ON CT SCANNING}

In this new era of radiology, CT scanning has become the primary method of assessing head injury since its inception, and further it can be supplemented by cervical spine radiograph (lateral view) [4,15-18].

Previously in the studies conducted early there was a direct relation between the severity of clinical symptoms and the demonstration of abnormalities [8], whereas in this study all the cases show one or more post-traumatic sequel, indicating a considerable severity, and up to $60 \%$ of this study showed two or even three intracranial sequelae, such as an epidural, and an intracerebral hematomas, or a subarachnoid, a subdural and an intracerebral 
hematomas all together as showed above in Figure 1. The incidence of abnormal CT findings are variable in different series ranging from $37 \%, 73 \%, 86 \%$ to $98 \%$ [7, 19,20].

\subsection{Diffuse Brain Swelling (DBS) (Figure 3)}

DBS occurs when there is loss of cerebral autoregulation which results in increase in blood flow and blood volume forcing CSF out of the ventricles and subarachnoid spaces, which causes compression of cistern spaces and ventricles which presents as mild increased density of white matter [4,20,21-24].

DBS is observed more among pediatric cases following head trauma, and it is usually due to a rapid hyperemia, since children differ in mode of injury, flexibility due to presence of sutures and poor myelination and in a rapid response to trauma in the form of vasodilatation and increase in cerebral blood flow [4,5,25-27].

It is observed that DBS is more common among children following head injury because of various reasons like due to unossified sutures there is flexibility, myelination is poor, they don't sustain injury in the same way as adults and the response to trauma is rapid in the form of hyperemia and vasodilatation $[4,6]$.

\subsection{Localized Brain Edema (LBO)}

LBO is seen as an ill-defined hypodense area with shift of the midline structures to the contralateral side which may be the only presenting feature [12,27-29].

Its high incidence can be explained by it being usually accompanied with other sequelae.

\subsection{Intracerebral Hematoma (ICH) and Brain Contusion (Figure 4, Arrow 1)}

Brain contusions can be hemorrhagic and non hemorrhagic and they are commonly found in patients who sustained blunt head trauma and in acceleration and deceleration trauma where they present as coup or contre coup injuries [21]. Early detection of contusion in patients who belong to the following group i.e., Anisocoria, low GCS scores, trauma due to fall or older patients $(>60)$ years.

They will be benefited from prompt neurosurgical intervention [22].

On plain CT, contusions appear as hypodense areas if hemorrhage is absent and hyperdense areas if hemorrhage is present. As the clot signal is similar to the brain parenchyma in MRI in the acute stages of head trauma, CT is more sensitive in detecting clots within 24 hours of injury than MRI [23].

ICH appears on CT as a well-defined hyperdense area surrounded by a hypodense area of perilesional edema. According to few studies both ICH and contusions si-

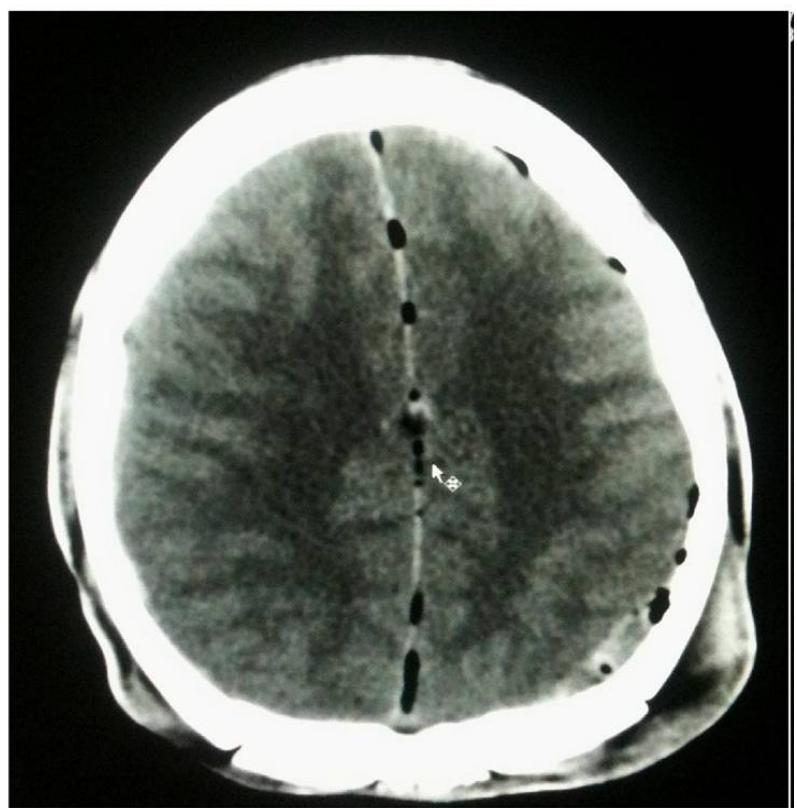

Figure 3. Diffuse Brain Swelling (DBS).

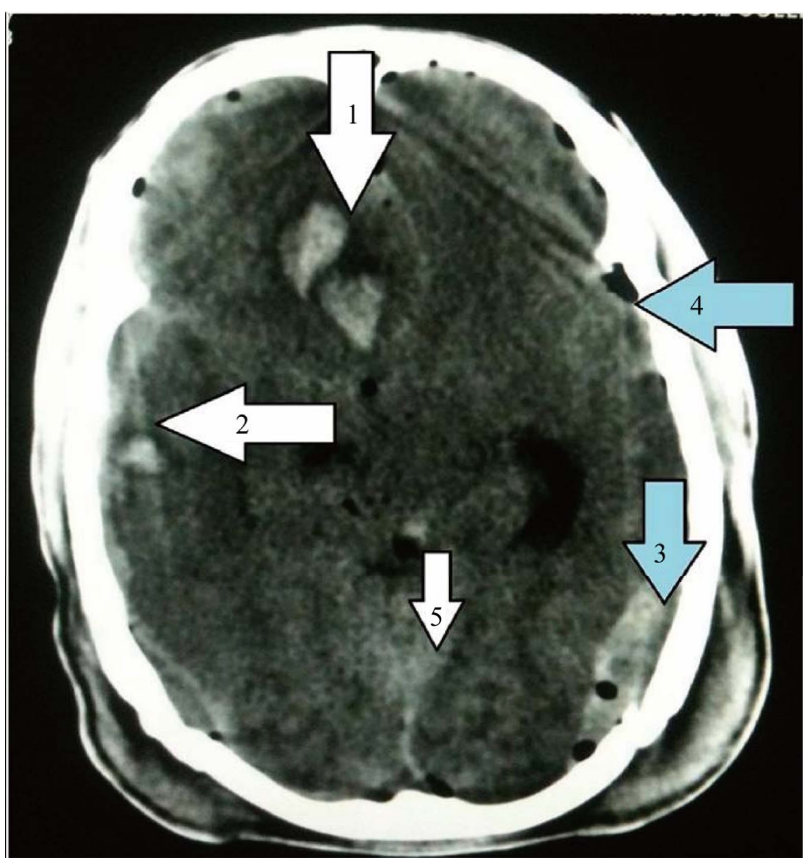

Figure 4. Arrow 1: Intracerebral Hematoma (ICH) and brain contusion; arrow 2: Acute Subdural Hematoma (ASD); arrow 3: Epidural Hematoma; arrow 4: pneumocephalus; arrow 5: Subarachnoid Hematoma (SAH).

multaneously exist in the same case with ICH occurring more commonly and is more frequently associated with other post traumatic consequences [7].

This study documented a little high incidence of contusions, and ICH of $22 \%$ and $20 \%$, respectively, where the previous reports declared low $[2,3,6]$, this might reflect the severity, and the selectivity of the cases of head 
traumas included in this study. In another study the incidence of contusion and ICH were $13 \%$ and $25 \%$ respectively [7].

According to Holburn's force shear theory countercoup injury which occurs on the opposite side of injury can be explained on the basis of rotational acceleration leading to shear force strains [10-12].

\subsection{Acute Subdural Hematoma (ASD) (Figure 4, Arrow 3)}

Subdural hematomas which are more common in patients with head injury are evaluated using subdural CT windows (i.e., wider soft tissue windows) as they compensate for the partial volume averaging in CT [22].

Extraaxial concavo-convex hyperdense area with a concave inner margin and convex outer margin and blood fluid level are occasionally seen. Mass effect with compression of adjacent cortical sulci and ventricles and effacement of adjacent cisterns, midline shift can be seen due to it. It can also present as iso or hyperdense area and can occur in interhemispheric fissure especially along postero-superior aspect in head trauma cases $[8,11]$. Acute SDH is found in $18 \%$ of this study (Figure 2), other studies have shown 19\%, 9\%, 12.6\%, 16\% and $59 \%$ [7]. A subdural hygroma appear as a peripheral, extra axial semilunar collection with convex outer border and concave inner border equal in density to CSF, and it is difficult to distinguish it from a chronic SDH on CT scanning, on the other hand it can be fatal and may have the same clinical significance as a subdural hematoma [7, 15,25-29].

\subsection{Epidural Hematoma}

An EDH appears as an extra-cerebral biconvex hyperdense elliptical collection with a sharply defined edge, and it arises within the potential space between the skull and dura and usually associated with skull fracture (75\% - 95\% of cases). Most commonly bleeding is from an injured middle meningeal artery, near to the inner table, from a fracture of the adjacent bony cranium with shift of ventricles $[7,14,25,29]$.

\subsection{Subarachnoid Hematoma (SAH) (Figure 4, Arrow 5)}

Subarachnoid hemorrhage is often seen adjacent to a contusion and occurs in children and elders who have large subarachnoid spaces. CT is more accurate in detecting acute SAH than MRI Because the blood in acute SAH has a low deoxyhemoglobin, which appears similar to brain parenchyma on T1- and T2-weighted spin echo images [24,25]. It occurs due to injury of small arteries and veins on the surface of brain and on CT appears as a hyperdensity in the sylvian fissure, sulci, basal cisterns or in the interhemispheric fissure. Care should be taken to not to misinterpret the calcified falx in the elderly as SAH.

A SAH over the hemispheres is due to fracture, local haematoma or contusion whilst a SAH is usually found isolated in basal cistern with a fractured base of skull $[7,8,15,16]$.

With increasing severity of the head trauma incidence of SAH increases which explains the wide range of incidences in different series [9].

\subsection{Diffuse Axonal Injury}

DAI presents diffuse neuronal injury. It is referred to as "shearing injury". Causes being acceleration, deceleration and rotational forces which result in portions of the brain with different densities to move relative to each other resulting in the deformation of axons and disruption of axoplasmic transport [18]. Immediate loss of consciousness is typical of these injuries. Despite the patient's presentation with a profound neurological deficit, CT can be normal. DAI is diffuse and bilateral, appears on CT as multiple foci of high attenuation of $5-15 \mathrm{~mm}$ involving the lobar white matter at the gray-white mater interface most commonly, corpus callosum, dorsal lateral upper brain stem, the basal ganglia or in the posterior limb of internal capsule however, others add brain swelling and intraventricular hematoma too [28,29]. DAI denotes a poor prognosis $[1,8,11]$, CT is not sensitive in detecting DAI as most of the lesions are small and non hemorrhagic which can be missed $[8,17,18]$.

\subsection{Intraventricular Hematoma}

Traumatic intraventricular hemorrhage can present as associated with other post traumatic sequelae which occur in patients with severe head trauma such as DAI, gray matter injury and brain stem contusion. Therefore its presence indicates severe head trauma which is an ominous sign $[3,7]$.

\subsection{Fractures}

CT is the imaging modality of choice for detecting fractures and depending on their location, type prompt surgical intervention can be done in order to prevent CSF leakage, infection, haemorrhage or vascular compromise choice. Surgical elevation of open skull fractures which are depressed more than the full thickness of the skull should be done [26]. To exclude pneumocephalus all patients with skull base fractures should receive a follow up CT scan [27].

\subsection{Pneumocephalus and/or Hydrocephalus}

A pneumocephalus (Figure 4, arrow 4) presents as areas 
of low attenuation on CT (air pockets) and can result from fracture of any air containing cavity like sinuses, mastoid bone and skull base $[7,14]$.

Hydrocephalus indicates obstruction anywhere along the CSF pathway because of any reason like blood clot in ventricles or extrinsic compression due to gross cerebral edema $[1,8,19]$.

\section{CONCLUSION}

Various signs and symptoms like age of patient, Glasgow coma scale, vitals of patient and pupillary reaction were all shown to be significant predictors of patient condition and prognosis after head injury. Apart from this in the acute emergency situation, CT scan brain is a classical radiological modality to define status of head injury patient and very helpful to treat the head injury patients in golden hours without wasting more time.

\section{REFERENCES}

[1] Hidayat, S. (1989) Acute head trauma, an evaluation by CT scanning and conventional radiology. DMRD Dissertation, College of Medicine, Mosul University, Mosul.

[2] Hagga, J.R., Lanzieri, C.F. and Gilkeson, R.C. (2001) CT and MRI imaging of the whole body. Mosby.

[3] Quayle, K.S., Quayle, K.S., Jaffe, D.M., Kuppermann, N., Kaufman, B.A., Lee, B.C., et al. (1997) Diagnostic testing for acute head injury in children: When are head CT and head radiographs indicated? Pediatrics, 99, 11. doi:10.1542/peds.99.5.e11

[4] Hydel, M.L., Preston, C.A., Mills, T.J., Luber, S., Blaudean, E. and Deblielux, M.C. (2000) Indications for computerized tomography in patients with minor head injury. The New England Journal of Medicine, 343, 100-105. doi:10.1056/NEJM200007133430204

[5] Zimmerman, R.A., Bilaniuk, L.T., Genneralli, T., Bruce, D., Dolinskas, C. and Uzzell, B. (1978) Cranial CT in diagnosis and management of acute head trauma. American Journal of Roentgenology, 131, 27-34. doi:10.2214/ajr.131.1.27

[6] Norlund, A., Marke, L.A., Geijerstam, J.L., Ordoson, S. and Britton, M. (2006) OCTOPUS study investigators. Immediate CT scanning or admission after mild head injury: Cost comparison in randomized controlled trail. $B M J$, 333, 469. doi:10.1136/bmj.38918.659120.4F

[7] Geijerstam, J.L., Ordsson, S. and Britton, M. (2006) Octopous study investigators. Medical outcome after immediate computerized tomography or admission for observation in patients with mild head injury: Randomize controlled trail. BMJ, 333, 465. doi:10.1136/bmj.38918.669317.4F

[8] Youmans, J.R. (1982) Neurological surgery. Saunders, Philadelphia.

[9] Khalili, A.H. (1988) The value of a skull XR in the early management of head injury. Postgrad Doctor Middle East, 11, 3891-3893.
[10] Sutton, D. (2003) Textbook of radiology and imaging. 7th Edition, Curchill Livingstone.

[11] Swischuk, L.E. (1997) Imaging of the newborn, infant, and young child. 4th Edition, Lippincott Wiliams \& Wilkins.

[12] Zimmerman, R.A., Gibby, W.A. and Carmody, R.F. (2000) Neuroimaging, clinical and physical principles. SpringerVerley, New York.

[13] Tuny, G.A., Kumar, A., Richardson, R.C., Jenny, C. and Brown, D.B. (2006) Comparison of incidental and non incidental traumatic head injury in children on non-contrast computerized tomography. Pediatrics, 118, 626-633. doi:10.1542/peds.2006-0130

[14] Hammoud, D.A. and Wasserman, B.A. (2002) Diffuse axonal injuries: Pathophysiology and imaging. Neuroimaging Clinics of North America, 12, 205-216. doi:10.1016/S1052-5149(02)00011-4

[15] Koo, A.H. and La Rouge, R.L. (1977) Evaluation of head trauma by CT. Radiology, 123, 334-350.

[16] French, B.N. and Dublin, A.B. (1977) The value of CT scanning in the management of 1000 consecutive cases of head injuries. Radiology, 125, 464.

[17] Al-rawi, W., Ameen, A. and Altaee, M. (1995) Computerized tomographic scan findings with persistent acute posttraumatic headache. Basrah Journal of Surgery, 1, 74-78.

[18] Armstrong, P. and Wastie, M.L. (2001) A concise textbook of radiology. Arnold.

[19] Stark, D.D. and Bradely, W.G. (1995) Magnetic resonance imaging. In: Sosin, D.M., Sniezek, J.E., Waxweiler, R.J., Eds., Trends in death associated with traumatic brain injury, 1979 through 1992. Success and Failure. JAMA, 273, 1778-1780.

[20] Sosin, D.M., Sniezek, J.E. and Thurman, D.J. (1996) Incidence of mild and moderate brain injury in the United States. Brain Injection, 10, 47-54. doi:10.1080/026990596124719

[21] Traumatic Brain Injury (1997) Colorado, Missouri, Oklahoma, and Utah, 1990-1993. Morbidity and Mortality Weekly Report, 46, 8-11.

[22] SINGH, SURYAPRATAP (2009) An unusual case of a compound depressed skull fracture. Journal of Surgery Pakistan (International), 14, 184-186.

[23] Gutman, M.B., Moulton, R.J., Sullivan, I., Hotz, G., Tucker, W.S. and Muller, P.J. (1992) Risk factors predicting operable intracranial hematomas in head injury. Journal of Neurosurgery, 77, 9-14. doi:10.3171/jns.1992.77.1.0009

[24] Bradley, W.G. (1993) MR appearance of hemorrhage in the brain. Radiology, 189, 15-26.

[25] Holmes, E.J., Forrest-Hay, A.C. and Rakesh, R.M. (2008) Fundamentals of CT imaging. In: Holmes, E.J., ForrestHay, A.C. and Misra, R.R., Eds., Interpretation of Emergency Head CT: A Practical Handbook, Cambridge University Press, Cambridge, 3-9.

[26] Ashikaga, R., Araki, Y., Ishida, O. (1997) MRI of head injury using FLAIR. Neuroradiology, 39, 239-242. 
doi:10.1007/s002340050401

[27] Gruen, P. (2002) Surgical management of head trauma. Neuroimaging Clinics of North America, 12, 339-343. doi:10.1016/S1052-5149(02)00013-8

[28] Zee, C.S., Hovanessian, A., Go, J.L. and Kim, P.E. (2002) Imaging of sequelae of head trauma. Neuroimaging Clinics of North America, 12, 325-338.
doi:10.1016/S1052-5149(02)00004-7

[29] Mittl, R.L., Grossman, R.I., Hiehle, J.F., Hurst, R.W., Kauder, D.R., Gennarelli, T.A., et al. (1994) Prevalence of MR evidence of diffuse axonal injury in patients with mild head injury and normal head CT findings. American Journal of Neuroradiology, 15, 1583-1589. 